The Egyptian Journal of Hospital Medicine (January 2019) Vol. 74 (4), Page 802-808

\title{
Awareness of heat-related illnesses in population of Saudi Arabia
}

\author{
Mohammad Aljumaan, Faisal Alhawaj, Saleh Alkhalifa, Najebah Alhussain, Ali Alhashim, \\ Layan Alahmadi, Fatimah Alkhunaizi, Saleh Aljarudi
}

Emergency Department, Imam Abdulrahman Bin Faisal University, Eastern Provence, Saudi Arabia

Correspondin author: Saleh Alkhalifa, E-mail: s.khalifa013@gmail.com, Mobile number: +966567101389

\begin{abstract}
:
Background: heat stroke is a preventable severe condition of heat-related illnesses; it is an emergency condition affecting millions of people around the world. Heat related illnesses ranged from mild heat exhaustion to life-threatening heat stroke. Early detection and treatment of heat-related illnesses crucial to avoid mortality and morbidity. The aim of this study was to evaluate awareness of heat illnesses, its symptoms, treatment and prevention among the population of Saudi Arabia.

Methodology: a cross-sectional study carried out an online survey, Saudi Arabia, between, 1 November 2017 and 23 June 2018. Data were collected by using a valid multiple-choice question (MCQ). Survey questions included the possible thought causes and risk factors of both heat stroke and heat exhaustion.

Result: a total of 865 participants, the majority were Saudi citizens (806). Age and gender were almost equal. 281 were involved in the medical fields. Conclusion: this study assessed the awareness of heat stroke and heat exhaustion among the health staff and medical students in different aspect of heat-related illness (Mainly heat stroke and heat exhaustion) and compared it to the level of awareness of general population in some aspect of heat-related illnesses. Almost, two third of medical personnel differentiate heat stroke definition when it was listed among other heat-related illnesses.in the other hand, knowledge of heat exhaustion definition was markedly lower among both health staff and non-health staff.44.6\% of the participants chose all cooling methods as appropriate treatment of heat stroke. $71.1 \%$ knows that heat stroke had more devastating outcomes if not managed properly and rapidly than heat exhaustion.34\% of the medical personnel thought avoiding hot spots was the best way to prevent heat-related illnesses; a similar number of the non-medical (31\%) also thought the same. Awareness of heat-related illnesses and differentiation between them in relation to the definition, sign, symptoms, diagnosis, and treatment is not satisfactory.
\end{abstract}

Keywords: heat stroke, heat exhaustion, heat-related illness, awareness of heat-related illness.

\section{INTRODUCTION}

Heat stroke is a life threating emergency under the category of heat related-illness which affect millions of people in the world ${ }^{(\mathbf{1}, 2)}$. Heat-related illnesses (HRIs) are common and preventable conditions that range from minor form (e.g. heat cramps) to life-threatening heat stroke ${ }^{(3)}$. Heat exhaustion is the milder form of Heat-related illnesses with a body temperature between $100.4 \mathrm{~F}$ $\left(38^{\circ} \mathrm{C}\right)$ and $104 \mathrm{~F}\left(40^{\circ} \mathrm{C}\right)$. Heat exhaustion is characterized by mild dehydration with or without sodium disturbance after excessive exercise, exposure to extremely high environmental temperature, chronic loss of volume, low fluid intake, and failure to adapt to surrounding temperature ${ }^{(3,4)}$. Symptoms of heat exhaustion included weakness, irritability, dizziness, vomiting, nausea, headache, diarrhea, goose flesh, headache and lose of coordination (1), Immediate management of Heat exhaustion includes hydration, move to a shaded and cool location, rest and monitor for resolution. If not treated heat exhaustion can complicate to a more sever condition called Heatstroke ${ }^{(2,5)}$. Heatstroke defined as a condition which body temperature raised to $104^{\circ} \mathrm{F}\left(40^{\circ} \mathrm{C}\right)$ or above with central nerves system dysfunction. Heat stroke manifested by confusion, dizziness, hallucination, delirium, seizures, tachycardia, hypotension and multi-organ dysfunction, which is the most serious complication that can lead to death ${ }^{(\mathbf{1 , 6})}$, an early 
differentiation between heat stroke and heat exhaustion is crucial to avoid morbidity and mortality. However, Heatstroke differs from heat exhaustion in three clinical manifestations. First, hyperthermia with body temperature more than 40.6C. Second, Central nervous system dysfunction conspicuous as delirium, convulsions, and coma. Third, it usually has anhydrosis which indicate thermoregulatory failure ${ }^{(7)}$. Heatstroke can be complicated by multi-organ dysfunction which includes: acute respiratory distress syndrome, rhabdomyolysis, intestinal ischemia and encephalopathy ${ }^{(\mathbf{4}, \mathbf{8})}$. In addition, dehydration and hemoconcentration may result in electrolytes imbalances such as hypercalcemia along with hyperproteinemia which eventually leads to acute renal failure and further increasing the risk of mortality ${ }^{(9)}$. A cross-sectional study was done in Karachi showed various in awareness of heat exhaustion between medical staff, the treatment is well known but there is a little confusion about its symptoms. However, the laymen need to be aware of heat exhaustion ${ }^{(\mathbf{1 0})}$. Another study was done and the conclusion was: the awareness of heat illness was clear among participants and there are aware of heat stroke as a dangerous form of heat stress, however most of them did not understand how much heat stroke can be dangerous, furthermore, 1 out of 5 participant does not has clear understanding of the idea of heat stress as a part of heat illnesses ${ }^{(\mathbf{9})}$. Furthermore, a study done among NYC population showed $30 \%$ did not know about the seriousness of hot weather warnings in 2011, even those who knew about the warnings they don't appraise themselves to be at danger or think of $\mathrm{AC}$ as a protection from the hot wave ${ }^{(3)}$.

There was a lack of studies done in Saudi Arabia that evaluated the awareness of the population about heat exhaustion. This study aimed to evaluate awareness of heat exhaustion's symptoms, treatment, and prevention among the population of Saudi Arabia.

\section{Methodology}

This cross sectional study was carried out online survey, Saudi Arabia, between 1 November 2017 and 23 June, 2018. Data were collected by using a valid multiple-choice questions (MCQ) were distributed containing 8 questions and 4 questions related to demographic characteristics. Survey questions included the possible thought causes and risk factors of both heat exhaustion and heat stroke. At the beginning of the questionnaire, each participant was asked to give his consent to participate in the study, and they were asked to answer MSQ question with only one answer unless otherwise indicated of more than one answers are possible. The questionnaire validated by using content validation in which three emergency medicine consultants participated and the validity index was 0.96. Questionnaire reliability was calculated using Cronbach alpha and the reliability index was 0.718 .

\section{Statistical analysis}

Data were entered in Microsoft Excel first then transferred to SPSS software version 23 .

\section{Result}

A total of 865 participants, gender almost equal; male $437(50.5 \%)$ and 428 (49.5\%), age were categorized to groups the majority was between 18-30 years, $722(83.5 \%)$ (Table 2), Saudi citizens were also the majority 806 (93.2\%).Regarding occupation student compromised 285 (32.9\%) and medical student of $153(17.7 \%)$ then who chose not to answer other 149 (17.2\%). Out of total 281 were medical fields, again gender, nationality and age were almost similar to the total participants 
Awareness of heat-related illnesses in population of Saudi Arabia

\begin{tabular}{||l|l|l|}
\hline TABLE 1: & \multicolumn{2}{l|}{} \\
\hline Participant's characteristics (All) & 865 & $(\%)$ \\
\hline Gender & & \\
\hline Male & 437 & 50.5 \\
\hline Female & 428 & 49.5 \\
\hline Nationality & & \\
\hline Saudi & 806 & 93.2 \\
\hline Non-Saudi & 59 & 6.8 \\
\hline Age & & \\
\hline $18-30$ & 722 & 83.5 \\
\hline $31-40$ & 75 & 8.7 \\
\hline $41-50$ & 48 & 5.5 \\
\hline $50+$ & 20 & 2.3 \\
\hline Occupation & & \\
\hline Student & 285 & 32.9 \\
\hline Medical student & 153 & 17.7 \\
\hline Health staff include (doctors, nurses...etc.) & 128 & 14.8 \\
\hline Military & & \\
\hline Outdoor worker & 4 & 0.5 \\
\hline Office worker & 56 & 6.5 \\
\hline Other & 90 & 10.4 \\
\hline & 149 & 17.2 \\
\hline
\end{tabular}

\begin{tabular}{||l|l|l|}
\hline \multicolumn{2}{||}{ TABLE II } & \multicolumn{2}{l|}{} \\
\hline Participant's characteristics (Medical only) & $\begin{array}{l}\text { N } \\
281\end{array}$ & \\
\hline Gender & & \\
\hline Male & 134 & 47.7 \\
\hline Female & 147 & 52.3 \\
\hline Nationality & & \\
\hline Saudi & & \\
\hline Non-Saudi & 267 & 95.0 \\
\hline Age & 14 & 5.0 \\
\hline $18-30$ & 4 & 1.4 \\
\hline $31-40$ & 257 & 91.5 \\
\hline $41-50$ & 17 & 6.0 \\
\hline $50+$ & 3 & 1.1 \\
\hline
\end{tabular}


Mohammad Aljumaan et al.

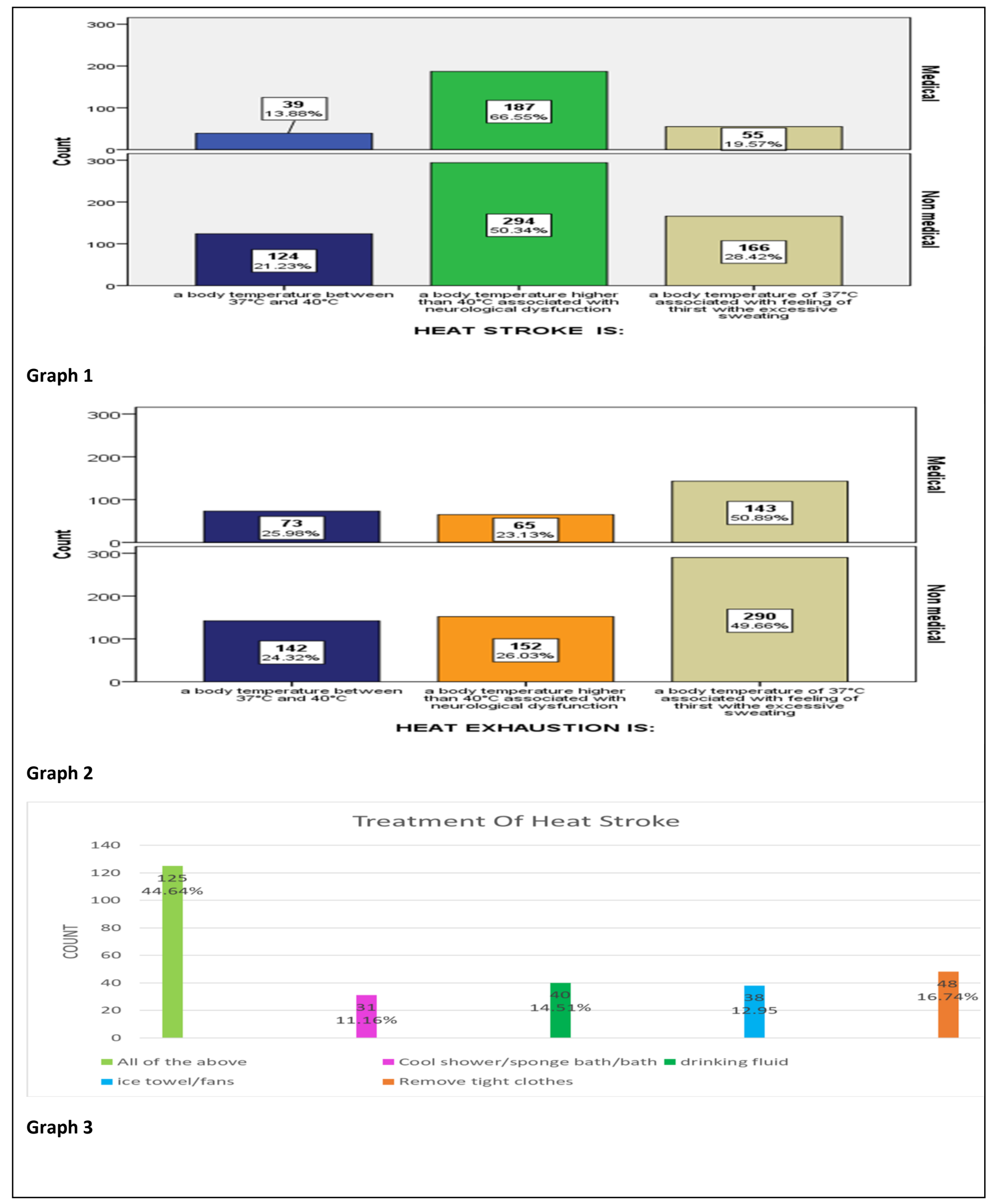




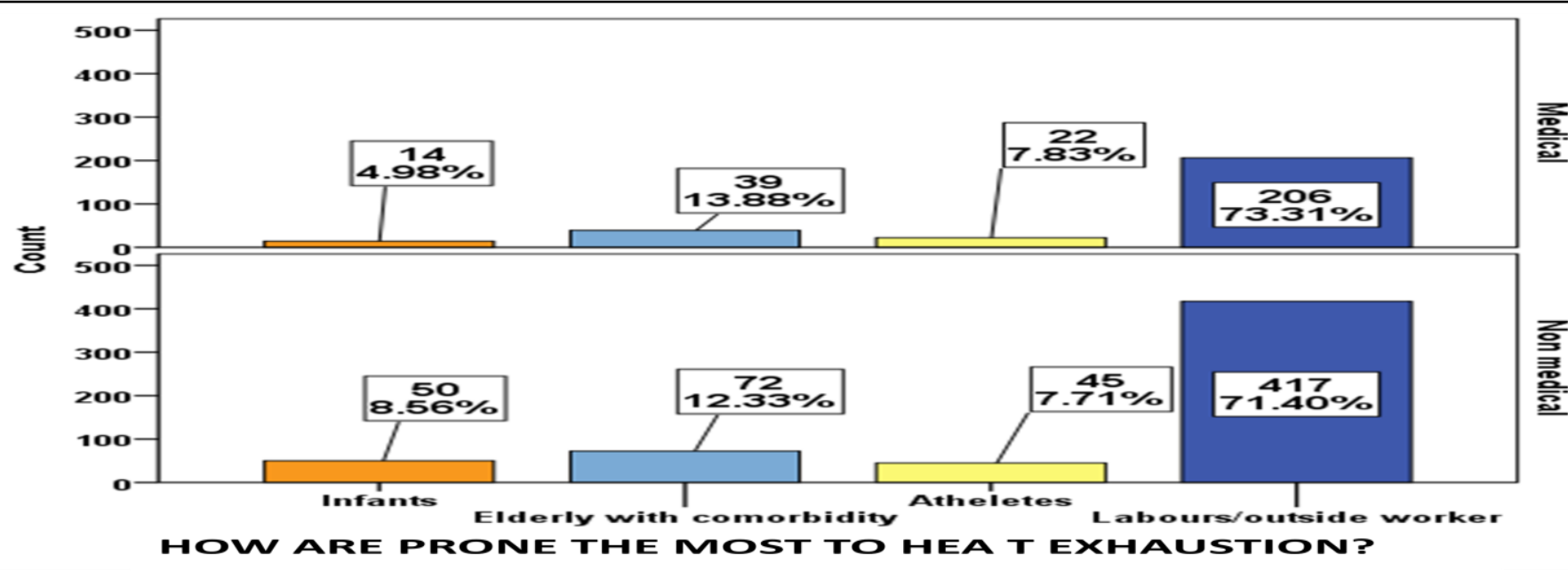

Graph 4

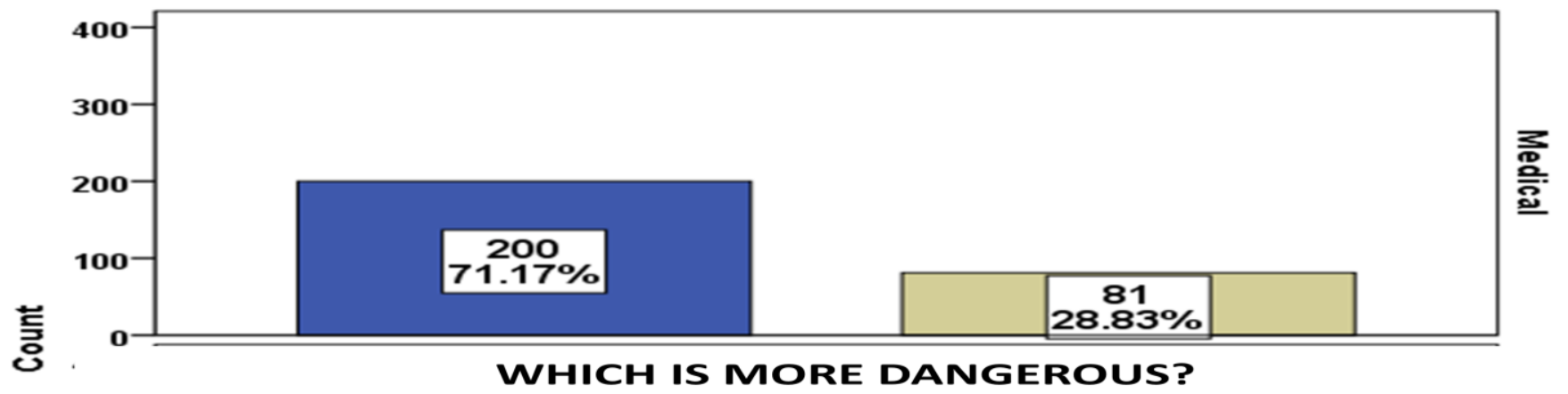

Graph 5

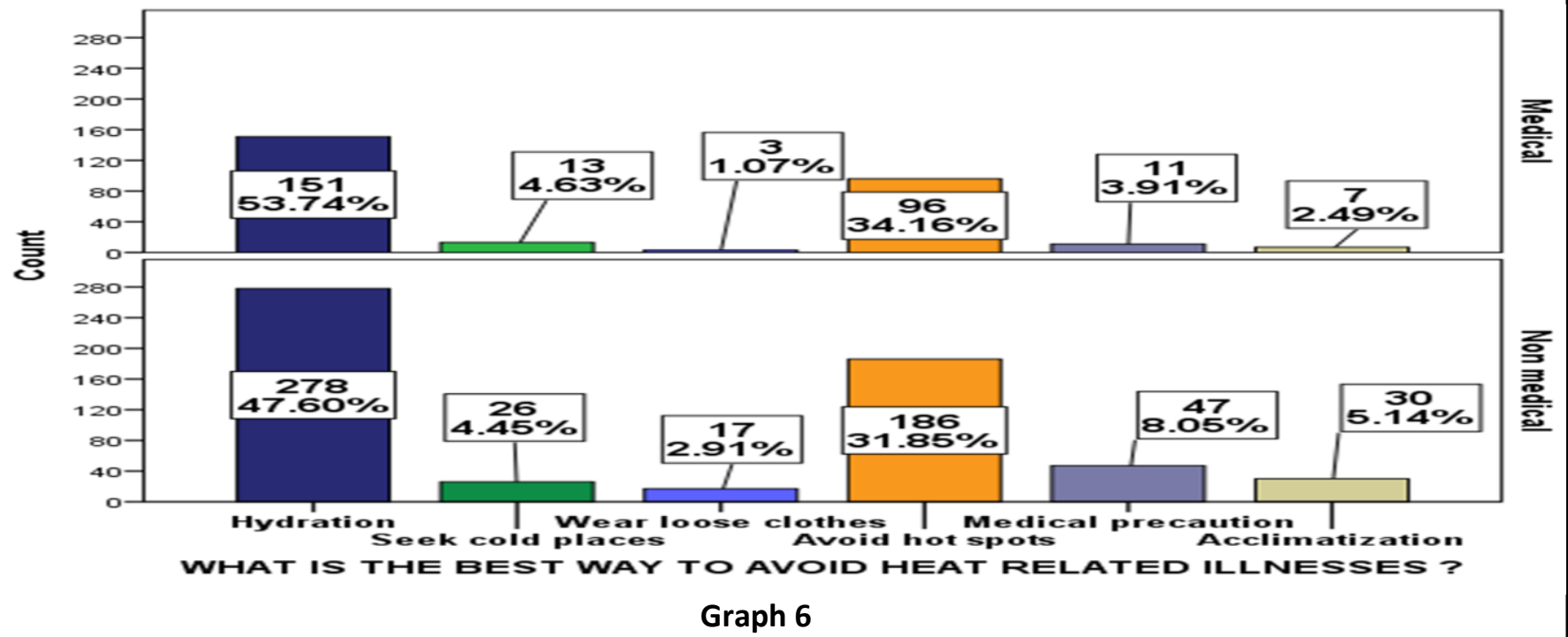




\section{Discussion}

This study assessed the awareness of heat stroke and heat exhaustion among health staff and medical students in a different aspect of heatrelated illness (mainly heat stroke and heat exhaustion), and compare it to the level of awareness of general population in some aspect of heat-related illnesses.

Graph 1 shows the that almost two-thirds of medical personnel differentiate the definition of heat stroke when it was listed among the definition of other heat-related illnesses, in the other hand only half of the participants recognize about heat stroke definition ${ }^{(6,12,13,14)}$.

In contrast to heat stroke definition, knowledge of heat exhaustion definition was markedly lower among both health staff and non-health staff which it was almost the same, 25.98\%, $24.32 \%$ respectively. As illustrated in graph $2^{(15)}$.

According the treatment of the heat stroke, the purpose of the initial treatment is to rapidly decreasing core temperature ${ }^{(\mathbf{1})}$. This may be started in the field (e.g., by external cooling) before a definitive diagnosis is made. After transfer to a medical facility, cooling should be continued. Cooling methods may be either external or internal; external methods are preferred (16,17). When available, intravenous fluid such as normal saline should be given. Studies showed that antipyretics are not effective in treating heat stroke and their use is not recommended ${ }^{(18)}$. Graph 3 shows $44.6 \%$ of the participants chose all the listed methods as an appropriate method of treatment of heat stroke. According to the best way of treatment of heat stroke, multiple studies agreed that cooling shower and bath the best method ${ }^{(\mathbf{1 9})}$, they were the least to be chosen between other methods.

Certain factors increase the susceptibility of the population to develop heatstroke. Pre-existing comorbidities subject the individual to the higher risk of developing heat stroke as they impair the thermoregulatory system in the body. Occupations involving performing a high level of physical activities in a hot weather such as soldiers and athletes can also predispose them to heat stroke ${ }^{\text {(20). }}$. The local climate is considered a risk factor as it determines the level of individuals' acclimatization and therefore their sensitivity to rising temperature ${ }^{(8)}$. Majority of population and health staff consider outside workers and labors who get exposed to the sunlight most of their time during work are the most types of people at risk of heat stroke as shown in graph 4 where only $13.8 \%$ of the medical and $12.3 \%$ of the nonmedical consider elderly with comorbidity are more prone than others to have heat exhaustion, even though epidemiological studies showed that Age is an important risk factor as elderly and children are more vulnerable to heat stroke than adults as their ability to regulate body temperature is limited ${ }^{(8)}$.

Graph 5 shows that in despite the limited knowledge of heat stroke and heat exhaustion definition is low, $71.1 \%$ knows that heat stroke has more devastating outcomes if not managed properly and rapidly than heat exhaustion ${ }^{(\mathbf{2 1 , 2 2})}$.

Prevention methods are very functional in decreasing mortality and morbidity associated with heat-related illnesses ${ }^{(23)}$. People who at high risk of heat illnesses should be advised to increase fluid intake to maintain hydration, avoid sun exposure, wear loose, light clothing, and monitor their exertion level. Athletes should be instructed to acclimatize for at least 3 to 4 days before exercising in high temperature climate. Heat injury can prompt inflammation that may increase risk on consequent days, patients most avoid the exposure to heat for 24 to 48 hours after a mild injury ${ }^{(23)}$. All these methods mentioned in graph 6 can be used to prevent heat-related illnesses but the most effective way is to avoid being in hot places from the beginning ${ }^{(24)}$, In graph 6,34\% of the medical personnel thought avoiding hot spots is the best way to prevent the medical consequences of heat, similar number of the non-medical (31\%) also thought the same.

\section{CONCLUSION}

This study goal was to assess the level of awareness of heat-related illness more precisely heat exhaustion and heat stroke on the aspects of the definition of heat stroke and heat exhaustion Group of people at high risk, treatment and Prevention. Our study showed how deficient the knowledge about the concept of heat-related illness among both health personnel including medical students and the general population. We suggest that more education need to be implemented in schools, colleges and pre-occupational courses concerning this subject and Moreover education 
programs needed among health staff.

\section{ACKNOWLEDGEMENT}

Authors would like to thank Dr Mohammad Alshahrani, Dr Thamir Aljunaid, and Dr Nasreen Maghraby for their contribution in validation of the questionnaire.

\section{REFERENCES}

1- Bouchama A, James P (2005):Heat stroke. New England journal of medicine, 346: 1978-1988.

2- GlazerJ (2005):Management of heatstroke and heat exhaustion. Am Fam Physician, 71: 21332140.

3- Becker, Jonathan A, Lynsey K (2011): Heatrelated illness.American family physician, 83:11 .

4- Nicholas D, Peterkin, MD Joseph S (2016): What is the best practice for the treatment of exertional heat illnesses (heat cramps, heat syncope, heat exhaustion, and exertional heat stroke). Athletic Training and Sports Health Care, 97:99.

5- Crowe J, Nilsson M, Kjellstrom T, Wesseling C (2015): Heat-Related symptoms in sugarcane harvesters. American journal of industrial medicine, 54:514-518.

6- Davis C, Tillman H, Chung T, Stravitz R, Reddy R, Fontana R (2017): Heat stroke leading to acute liver injury \& failure: A case series from the Acute Liver Failure Study Group. Liver International, 53:509-513.

7- Grubenhoff, Joseph A, Kelley F, Genie R (2007): Heat-related illness. Clinical Pediatric Emergency Medicine, 44:59-64.

8- Hifumi T, Kondo Y, Shimazaki J, Oda Y, Shiraishi S, Wakasugi M (2018): Prognostic significance of disseminated intravascular coagulation in patients with heat stroke in a nationwide registry. Journal of critical care, 44: 306-311.

9- Derek S, Alexander S, Lorentzson L, McCarty A (2010): Knowledge and awareness of heat-related morbidity among adult recreational endurance athletes. International journal of biometeorology, 54(4): 441-448.

10- Sarfaraz S, Karim S, Hussain H, Fatima W, Bano T, Sabir A (2015): Awareness regarding heat exhaustion; a survey based study in population of Karachi. European journal for pharmaceutical and medical research, 13:17.
11- Lane K, Wheeler K, Charles-Guzman K, Ahmed M, Blum M, Gregory K (2014): Extreme heat awareness and protective behaviors in New York City. Journal of urban health, 91(3): 403-414. 12- Wasserman D, Healy $M$ (2017): EMS Methods To Cool A Patient In The Field. https://www.ncbi.nlm.nih.gov/books/NBK459303/

13- Cheshire Jr, William $P$ (2016): Thermoregulatory disorders and illness related to heat and cold stress. Autonomic Neuroscience, 196: 91-104.

14- Alzeer H, Wissler H (2018): Theoretical analysis of evaporative cooling of classic heat stroke patients. International journal of biometeorology, 1:8.

15- Grogan H, Hopkins M (2002): Heat stroke: implications for critical care and anaesthesia. British journal of anaesthesia, 88(5): 700-707.

16- Harker J, \& Gibson P (1995): Heat-stroke: a review of rapid cooling techniques. Intensive and Critical Care Nursing, 11(4): 198-202.

17- Hadad E, Rav-Acha M, Heled Y, Epstein Y, Moran S (2004): Heat stroke. Sports Medicine, 34(8): 501-511.

18- Hassanein T, Razack A, Gavaler S, Van Thiel H (1992): Heatstroke: its clinical and pathological presentation, with particular attention to the liver. American Journal of Gastroenterology, 87: 1382-1389

19- Eichner R, (1998): Treatment of suspected heat illness. International Journal of Sports Medicine, 19(S 2): S150-S153.

20- Leon R, Helwig G (2010): Heat stroke: role of the systemic inflammatory response. Journal of applied physiology, 109(6): 1980-1988.

21- Luber G, McGeehin M (2008): Climate change and extreme heat events. American journal of preventive medicine, 35(5): 429-435.

22- Donoghue M, Sinclair J, Bates P (2000): Heat exhaustion in a deep underground metalliferous mine. Occupational and environmental medicine, 57(3): 165-174.

23- Nakai S, Itoh T, Morimoto T (1999): Deaths from heat-stroke in Japan: 1968-1994. International journal of biometeorology, 43(3): 124-127.

24- Weinberger R, Zanobetti A, Schwartz J, Wellenius A (2018): Effectiveness of National Weather Service heat alerts in preventing mortality in 20 US cities. Environment international, 116: 30-38. 